\title{
Molecular Characteristics and Phylogenetic Analysis of Short Beak and Dwarfism Syndrome- Related Novel Goose Parvovirus
}

\section{Yonglin Li}

Yangzhou University

Jingyu Jia

Yangzhou University

Qingling Mi

Yangzhou University

Yufeng Li

Shandong Academy of Agricultural Sciences

\section{Yuehua Gao}

Shandong Academy of Agricultural Sciences

\section{Guoqiang Zhu}

Yangzhou University

Jianye Wang ( $\boldsymbol{\nabla}$ wangjy@yzu.edu.cn )

College of Veterinary Medicine of Yangzhou University https://orcid.org/0000-0001-7173-566X

\section{Research Article}

Keywords: short beak and dwarfism syndrome, goose parvovirus, molecular characteristics, medium egg lethal dose, inverted terminal repeats, phylogenetic tree

Posted Date: March 23rd, 2021

DOl: https://doi.org/10.21203/rs.3.rs-349163/v1

License: (c) (1) This work is licensed under a Creative Commons Attribution 4.0 International License.

Read Full License

Version of Record: A version of this preprint was published at Archives of Virology on July 7th, 2021. See the published version at https://doi.org/10.1007/s00705-021-05145-4. 


\section{Abstract}

Short beak and dwarfism syndrome (SBDS) emerged in cherry valley duck flocks in China in 2015, and novel goose parvovirus (NGPV) was proved to be the etiological agent of SBDS. To date, whether SBDSrelated NGPV isolates possess common molecular characteristics remains unknown. In this study, three new NGPV strains (namely, SDHT16, SDJN19, and SDLC19) were isolated from diseased ducks showing typical SBDS and successfully passaged in embryonated goose or cherry valley duck embryo. The whole genomes of three NGPV strains shared $98.9 \%-99.7 \%$ homologies between each other but showed slightly lower homologies (95.2\%-96.1\%) with the classical GPV strains. A total of 16 common amino acid point mutations were produced in the VP1 proteins of six NGPV strains (SDHT16, SDJN19, SDLC19, QH, JS1, and SDLC01) compared with the classical Chinese GPV strains, among which nine amino acid sites were identical to the European GPV strain B. The non-structural protein Rep1 of the six NGPV strains generated 12 common amino acid mutations compared with the classical GPV strains. The phylogenetic analysis indicated that the Chinese NGPV strains clustered with the European SBDS-related NGPV strains, forming a separate branch, distinct from the group formed by the classical GPV strains. Taken together, the present study unveils the common molecular characteristics of the NGPV isolates and directs the conclusion that the Chinese NGPV isolates probably originate from a common ancestor with the European SBDS-related NGPV.

\section{Introduction}

Short beak and dwarfism syndrome (SBDS) emerged in cherry valley duck flocks in China in 2015 with the morbidity rate ranging from $5-25 \%[1,2,11]$. Ducks with this disease are characterized by a short beak, protruding tone, fragile bone, and retarded growth $[2,12]$. The poor body weight gain and heterogeneity within the flock lead to economic losses to the duck meat industry. SBDS was first reported in the 1970s in France in mule ducks and also reported in Poland in 1995 [14].

The causative agent of SBDS is closely related to the classical goose parvovirus (GPV), tentatively named as novel GPV or GPV variant [20]. GPV and Muscovy duck parvovirus are classified in the genus of Dependoparvovirus in the Parvoviridae family [3]. The viral genome has a 5.1-kb single-stranded linear DNA with equal polarities encapsidated in the icosahedral capsid. The genome is flanked with identical inverted terminal repeats (ITRs) and contains two ORFs, the left encoding the Rep protein and the right encoding the capsid protein [21]. The Rep ORF produces the largest protein Rep1 and several lowmolecular-weight Rep proteins from spliced mRNAs, which are involved in genome replication, interaction with ITR, and modulation of the downstream P41 promoter $[10,13,16,18]$. By alternative mRNA splicing and protease cleavage, the right ORF produces three structural proteins (VP1, VP2, and VP3), which assemble the viral capsid with a ratio of 1:1:8 $[7,16]$.

The GPV DNA can be detected by PCR from the internal organs of diseased ducks showing SBDS; however, viral isolation from tissues (liver, spleen, and kidney) is not always successful [1,20]. Viral isolation can be successfully achieved in susceptible duck embryos through the inoculation of tissue 
homogenate. Blind passages in embryonated duck eggs are necessary to establish good adaption for the primary novel goose parvovirus (NGPV) isolates [1]. Since the outbreak of SBDS in China, several genome sequences of NGPV isolates have been sequenced $[1,12,20]$, but whether these isolates possess common molecular characteristics and similar propagation capability in avian embryos remains unclear. Moreover, the evolution correlation between the NGPV isolates and the classical GPV strains and the European SDBS-related GPV isolates remains to be elucidated.

In the present study, three NGPV strains were isolated from SBDS cases during disease outbreaks in cherry valley duck flocks between 2016 and 2019, and their genomes were sequenced. Sequence alignments revealed that all Chinese NGPV strains harbored common amino acid mutations in the coding proteins compared with the classical GPV strains. Moreover, two NGPV strains isolated in different years exhibited obviously differential propagation ability in embryonated duck embryos. Phylogenetic analysis indicated that the Chinese NGPV isolates probably originated from the European SBDS-related NGPV.

\section{Materials And Methods}

\section{Viral isolation and passage}

For the diseased cherry valley ducks showing typical SBDS, their internal organ tissues, such as liver, kidney, and spleen, were sampled between 2016 and 2019 in Shandong Province, China. The tissue samples were placed in sterile saline for homogenization. The homogenate was clarified by centrifugation at $3000 \times \mathrm{g}$, and the supernatant was transferred and passed through a filter with a 0.22 $\mu \mathrm{m}$ pore diameter. The filtrate was added with penicillin $(1000 \mathrm{IU} / \mathrm{mL})$ and streptomycin $(1000 \mu \mathrm{g} / \mathrm{mL})$ and then used to inoculate susceptible 9-day-old embryonated goose eggs via the chorioallantoic membrane route. The eggs were incubated at $38^{\circ} \mathrm{C}$ for 12 days and candled three times every day. The dead embryos were placed at $4{ }^{\circ} \mathrm{C}$ for $6 \mathrm{~h}$, and then the allantoic fluid was pooled. The embryos were further examined for pathogenic lesions. The first-generation allantoic fluid containing virus was 1:10 diluted with sterile saline, and the dilution was used to inoculate another five 9-day-old goose eggs or cherry valley duck eggs for passage.

\section{Determination of medium egg lethal dose}

To evaluate whether the NGPV isolates isolated in 2016 and 2019 possess differential propagation capability in embryonated duck eggs, the medium embryo lethal dose $\left(E^{2} D_{50}\right)$ against two NGPV strains was calculated in accordance with the method of Reed and Müench [17]. Briefly, the viral stock in the form of allantoic fluid was diluted in sterile saline to give a 10 -fold dilution series between $10^{-1}$ and $10^{-7}$. For each dilution, $0.2 \mathrm{~mL}$ was inoculated into 9-day-old susceptible cherry valley duck eggs (five eggs every dilution) via the allantoic cavity route. The inoculated eggs were continuously incubated at $37^{\circ} \mathrm{C}$ and examined three times daily for seven days. The times of any embryo deaths were recorded by hours. The $\mathrm{ELD}_{50}$ experiments were repeated for three times and the mean value was calculated. 
Seven sets of primers were designed on the basis of the genomic sequence of the classical GPV strain LH (accession number KM272560, Table 1). These primers were used to amplify the overlapping fragments by using PrimeSTAR Max DNA polymerase (Takara, Dalian, China). Except for the 200-bp terminal fragments containing half of the ITR, five internal DNA fragments were gel-purified using the TIANgel Midi Purification Kit (Tiangen, Peiking, China) and then subjected to direct sequencing using the amplification primers. An " $A$ " tail was added to the 200-bp terminal fragments using the Taq DNA polymerase. The fragments were then ligated with the pMD19T vector (Takara, Dalian, China) and transformed into competent DH5a cells. Positive clones were screened for sequencing. The obtained sequences were combined to assemble the whole genome sequence using the SeqMan program packaged in Lasergene 7.0 (DNASTAR, Madison, USA).

In addition, the ITR nucleotide sequences of three strains were confirmed by sequence analysis of recombinant plasmids carrying the viral DNAs purified directly from the virions without PCR amplification. Virus purification and concentration from the allantoic fuid and extraction of genomic DNA were performed as previously described [19]. The extracted single-stranded DNA (sSDNA) was suspended in STE buffer (10 mM Tris, $1 \mathrm{mM}$ EDTA, $100 \mathrm{mM} \mathrm{NaCl}, \mathrm{pH}$ 8.0) and annealed by heating to $95^{\circ} \mathrm{C}$ for $5 \mathrm{~min}$ followed by slow cooling to $55^{\circ} \mathrm{C}$. The double-stranded DNA (dsDNA) was digested with Ncol, separated by electrophoresis, and purified using a gel extraction kit (Tiangen, Peking, China). The 1.3-kb DNA fragment containing the 3'-end ITR was ligated into the Ncol-Smal site of the pBSKNB plasmid [19] and used to transform competent cells of the Sure strain of E.coli. The positive clones were chosen for sequencing. To overcome difficulties in sequencing ITR regions with hairpin structure, the ITR was digested by Sphl, which cut the ITR in the middle loop region, and the resulting fragments were subcloned into the pUC18 plasmid for sequencing.

\section{Sequence alignment and phylogenetic analysis}

Fourteen strains, including six classical GPV strains and eight SBDS-related NGPV strains, were used in this study for sequence alignment and phylogenetic tree construction. JS1, QH15, and SDLC01 were three representative NGPV strains that were isolated at the early stage of the SBDS outbreak in China (Table 2). Except for D146/02 and D697/3/06, both of which are European SBDS-related NGPV isolates (Palya et al., 2009) and only have partial VP1 gene sequences deposited in GenBank, the 12 other strains have complete genome sequences available in GenBank. Homology comparisons of the coding proteins among the classical GPV strains and SBDS-related NGPV strains were performed using the MegAlign program packaged in Lasergene 7.0. Given that the European SBDS-related NGPV strains only have partial VP1 gene sequences (at the $N$ terminus) available in GenBank, the phylogenetic tree was constructed on the basis of a 427-bp VP1 gene fragment to explore the possible evolutionary origin of the Chinese SBDS-related NGPV strains. The trees were constructed using MEGA 7.0 [9] (Kumar et al., 2016) by the maximum likelihood model and bootstrap values were calculated based on 1000 replicates.

\section{Statistical analysis}


The two-tailed student's t-test was adopted to compare the mean death time (MDT) of duck embryos post-inoculation between the SDHT16 and SDJN19 isolate by using GraphPad Prism software version 6.01 (GraphPad Software Inc., San Diego, CA, USA) . P $<0.05$ was considered statistically significant. The data were expressed as mean \pm standard deviation (SD).

\section{Results}

\section{Viral isolation and passage in embryonated goose eggs}

Three organ tissue samples were collected from diseased cherry valley ducks showing typical SBDS between 2016 and 2019 in Shandong Province, China. The three samples were positive in PCR detection using specific GPV primers. Each sample was used to inoculate five susceptible 9-day-old embryonated goose eggs. The result showed that 1,2, and 1 of five embryonated goose eggs died between $234 \mathrm{~h}$ and $288 \mathrm{~h}$ post-inoculation. The three viral isolates were successfully passaged in another five 9-day-old embryonated goose eggs and killed all the goose embryos in a shorter time (between $112 \mathrm{~h}$ and $190 \mathrm{~h}$ ) post-inoculation. Hemorrhagic lesions in the head, neck, and legs typical of parvoviral infection were observed (Fig. 1). The three NGPV isolates were named SDHT16, SDJN19, and SDLC19.

\section{NGPV propagation in cherry valley duck embryos and ELD ${ }_{50}$ calculation}

Strain SDHT16 and SDJN19 were successfully passaged in 9-day-old embryonated cherry valley duck eggs (Fig. 1). Strain SDHT16 killed 16 duck embryos between $106 \mathrm{~h}$ and $197 \mathrm{~h}$ (averaging $150 \mathrm{~h}$ ) postinfection, and strain SDJN19 killed 16 duck embryos between $69 \mathrm{~h}$ and $163 \mathrm{~h}$ (averaging $104.6 \mathrm{~h}$ ). Statistical analysis indicated that the mean death time between the two NGPV strains was significantly different $(P<0.05)$ (Fig. 2).

Furthermore, the calculation of the medium lethal egg doses $\left(E L D_{50}\right)$ was performed in embryonated cherry valley duck eggs. The ELD 50 values of strain SDHT16 and SDJN19 reached $5 \times 10^{2.5} / \mathrm{mL}$ and $5 \times$ $10^{5.4} / \mathrm{mL}$, respectively, demonstrating that strain SDJN19 has a better propagation capability in embryonated cherry valley duck eggs than strain SDHT16.

\section{Genome sequencing and homology comparison}

The whole genomes of three NGPV isolates were amplified by PCR and sequenced. The genome of strain SDHT16 is 5050 bases long, which is four bases shorter than that of strain SDJN19 and SDLC19. Nucleotide deletions or insertions occurred in ITR with no exception. The three NGPV strains shared 98.9\%-99.7\% similarities at the genomic level but displayed slightly lower nucleotide homologies $(95.2 \%-96.1 \%)$ with the classical GPV strains (strain B, 82-0321, 06-0329, YZ99-6, and LH). The genome sequences of three NGPV strains were deposited in GenBank with accession numbers MN356043, MN356044, and MN356045. 
The Rep1 gene of the three NGPV strains (SDHT16, SDJN19, and SDLC19) was composed of 1884 nucleotides, coding 627 amino acids. Homology comparison indicated that the Rep 1 gene of three NGPV strains shared $99.3 \%-99.7 \%$ similarities between each other but displayed $96.0 \%-96.5 \%$ similarities with the Chinese GPV strains (82-0321, 06-0329, YZ99-6, and LH) and 96.8\% 96.9\% similarities with the European GPV strain B (Figure 3). Amino acid alignments among the six NGPV strains (SDHT16, SDJN19, SDLC19, SDLC01, JS1, and QH15) and the classical GPV strains indicated that 12 amino acid mutations were produced in the NGPV strains compared with the classical GPV strains (Table 3). The altered amino acid sites were I50T, K131R, A140S, A350V, V468I, E498R, R553K, N555T, E573Q/K, D594Y, N609D, and V617A.

\section{Sequence comparison of the VP1 proteins}

The VP1 gene of three isolates was composed of 2199 nucleotides, coding for 732 amino acids. Homology comparison indicated that the VP1 proteins of three NGPV strains shared $99.1 \%-99.7 \%$ nucleotide similarities between each other but showed $94.4 \%-95.1 \%$ similarities with the Chinese GPV strains (82-0321, 06-0329, YZ99-6, and LH) and 96.0\%-96.6\% similarities with the European GPV strain B (Figure 3).

Amino acid alignments of the VP1 proteins among the six NGPV strains (SDHT16, SDJN19, SDLC19, SDLC01, JS1, and QH15) and the classical GPV strains indicated that compared with the Chinese GPV strains, the six NGPV strains harbored 16 common amino acid alterations, among which nine mutated amino acid sites were identical with the European strain B (Table 4). The 16 altered amino acid sites were H28Q, T35S, A41D, Q89L, D114H, Q116H/Y, D142E, V144I, A180V, L207M, A/T210S, S450N, A524G, L537I, K575R, and D703N.

\section{Sequence comparison of ITRs}

The ITRs of three NGPV strains (SDHT16, SDJN19, and SDLC19) shared 98.1\%-100.0\% nucleotide sequence homologies but displayed $93.0 \%-94.0 \%$ homologies with the classical GPV strain LH. The alignments of ITR sequences revealed that strain SDHT16, SDJN19, and SDLC19 have additional two, four, and four nucleotide insertions in the stems of the palindrome region, respectively, compared with the classical GPV strain LH (Fig. 4).

\section{Phylogenetic tree construction}

Because only partial sequences of the European SBDS-related NGPV strains were sequenced, a 427-bp fragment at the $\mathrm{N}$ terminus of the VP1 gene was used to construct the phylogenetic tree. The results revealed that six Chinese SBDS-related NGPV, including SDHT16, SDJN19, SDLC19, SDLC01, JS1, and QH15, intimately clustered together and grouped with the European SBDS-related NGPV strains (D697/3/06 and D146/02), forming a separate branch (Fig. 5). The four Chinese GPV strains (LH, YZ99-6, 82-0321, and 06-0329) closely clustered together and grouped with two European GPV strains (VG32/1 and B), forming another separate branch. On the basis of the results of phylogenetic analysis, the 
Chinese SBDS-related NGPV probably evolved from a common ancestor with the European SBDS-related NGPV.

\section{Discussion}

Viral isolation and PCR characterization are the preferred methods of diagnosing SBDS cases. In contrast to the parvoviral disease of geese (Derzsy's disease), against which the GPV isolation is easily achieved when using embryonated goose eggs as the vector $[5,6,15]$, viral isolations against SBDS cases are not always successful $[1,14,20]$. The primarily isolated virus may be lost in the following blind passages in duck embryos; hence, the isolation rates of positive samples were comparatively low. In this study, goose embryos were used for viral isolation against SBDS cases. Although only a limited number of embryonated goose eggs died post-inoculation of positive samples, the primary isolates can stably kill the embryonated goose eggs in a shorter time in the next passaging stages. The results demonstrated that embryonated goose eggs still are a suitable platform for isolating NGPV from SBDS cases. However, it should be noted that classical GPV usually kills 12-day-old goose embryos between 3 and 7 days postinoculation of tissue sample from Derzsy's disease [6], implying that classical GPV possesses a better replication capability in goose embryos than NGPV.

Strain JS1, QH15, and SDLC01 were previously isolated by other teams in China in 2015 [1, 12, 20]. The isolation year of SDHT16 was closer to that of strain JS1, QH15, and SDLC01 than SDJN19 and SDLC19. The VP1 and Rep1 proteins of SDHT16 displayed 99.8\%-99.9\% nucleotide sequence identities with that of strain JS1, QH15, and SDLC01, but showed $99.1 \%-99.4 \%$ and $99.3 \%-99.5 \%$ nucleotide sequence identities with that of strain SDJN19 and SDLC19, respectively. The result demonstrated that strain SDHT16 has the closer relationship with the early NGPV isolates than the 2019 NGPV isolates.

Strain SDJN19 and SDLC19 shared the higher genomic sequence homology (99.8\%) than with SDHT16 (99.1\% and 99.0\%, respectively). In order to evaluate whether the 2019 NGPV isolates may have different replication ability from SDHT16, further comparisons were conducted between strain SDJN19 and SDHT16 in this study. The result demonstrated both strains can be passaged in embryonated cherry valley duck eggs, however, on the basis of the $E_{5 D_{50}}$ and MDT values comparisons, strain SDJN19 was found to possess a better propagation ability in embryonated cherry valley duck eggs than SDHT16. Given that the classical GPV cannot propagate in embryonated cherry valley duck eggs, we deduce that the minor nucleotide differences across the genome probably contribute to the differential propagation capability observed in strain SDJN19 and SDHT16. Whether strain SDJN19 and SDHT16 can exhibit the differential pathogenicity in cherry valley duck deserves to be investigated.

ITR acts as the replication origin of the genome, containing a number of transcription factor binding sites and short nucleotide repeats (TCCGGT) $[19,21]$. Compared with the classical GPV strain LH, nucleotide mutations were produced in the ITR region of three NGPV isolates. ITR sequences of two 2019 NGPV isolates (SDJN19 and SDLC19) shared 99.5\% similarities, but they showed a slightly low similarities (97.6\%-98.1\%) with SDHT16. Besides, three NGPV isolates harbored one or two additional nucleotide 
insertions in the stem region of ITR. However, all of these nucleotide mutations and insertions were found to be located in the base-pairing positions, hence the correct hairpin structure formation of ITR was not influenced. Altogether, the sequence comparison results of ITRs among three NGPV strains demonstrated a closer relationship between two 2019 NGPV isolates, which was in parallel with the comparison results of the coding proteins.

The characteristic amino acids sites belonging to the NGPV isolates were identified in this study. These site alterations produced in the NGPV strains played a key role in the host transition from goose to cherry valley duck. According to our observation, the amino acid alterations of both proteins were not evenly distributed in the peptide sequences. In terms of Rep 1 protein, 8 of 12 site alterations were located within one third of the peptide sequences adjacent to the carboxyl terminus. $C$ termini of the Rep1 protein contains the domain involved in transactivation of P41 promoter, which modulate the VP1 gene transcription [13]. For the VP1 protein, half of 16 alterations were located in the VP1u region of the VP1 protein. VP1 1 contains the phospholipase A2 (PLA2) domain, which is required for parvovirus entry and infectivity [8, 22]. Further analysis showed only an amino acid alteration (Q89L) was found to be located in the PLA2 motif (53-111 aa) of VP1u of GPV [22], other seven mutated sites in the VP1u lay outside of the PLA2 motif. Nevertheless, the amino acid residues outside the PLA2 motif are also important for maintaining a proper three dimensional structure that is essential for the PLA2 activity [4]. Together, the common amino acid mutations identified in this study should contribute to the host range shift of NGPV.

Sequence alignment and comparison revealed that the six Chinese NGPV isolates have closer relationship with the European strain B than with the Chinese GPV strains. In the phylogenetic tree, the Chinese NGPV isolates grouped with the European SBDS-related NGPV strains and formed a separate branch, distinct from the branch grouped by the classical GPV strains. The present analysis result indicates that the Chinese NGPV isolates probably originate from a common ancestor with the European counterpart. The European SBDS-related NGPV was isolated from diseased mule ducks, which are a sterile and intergenetic cross of Pekin and Muscovy ducks [14]. Therefore, whether a characteristic difference exists at the genome level between the European and Chinese SBDS-related NGPV isolates needs to be elucidated. Whole genome sequencing against the European SBDS-related NGPV isolates may be able to shed light on this matter.

Taken together, three new SBDS-related NGPV isolates were obtained in this study, and their genomes were sequenced. The differential propagation abilities in embryonated cherry valley duck eggs were observed between two NGPV strains isolated in different years. The characteristic amino acid differences between the NGPV strains and the classical GPV strains were identified, which should contribute to host range shift. Further phylogenetic analysis supported the conclusion that the Chinese NGPV isolates probably originate from a common ancestor with the European SBDS-related NGPV.

Last but not least, the VP1 protein of NGPV still shares almost 95\% amino acid identity with the classical GPV, irrespective of the characteristic amino acid differences. The structural proteins, including VP1, VP2, VP3, commonly constitute the protective antigens of GPV [7]. Hence, whether the traditional GPV vaccine 
currently in use against the Derzsy' disease can be applied in cherry valley ducks to prevent from SBDS deserves to be thoroughly investigated.

\section{Declarations}

\section{Funding}

This work was supported by the National Natural Science Foundation of China [grant numbers 31572551 , 31172317] and by a project funded by the Priority Academic Program Development of Jiangsu Higher Education Institutions.

\section{Conflicts of interest/Competing interests}

The authors declare that they have no competing interests.

\section{Ethics approval}

The procedure for inoculation of embryonated goose or duck eggs was approved by the Animal Care and Use Committee of Yangzhou University and performed in accordance with the "Guidelines for Experimental Animals" of the Ministry of Science and Technology (Beijing, China). No specific permissions were required for these locations/activities.

Consent to participate (include appropriate statements)

All the listed authors participated in this study with informed consents

Consent for publication (include appropriate statements)

Written informed consent for publication was obtained from all participants.

\section{Availability of data and material}

Not applicable

\section{Code availability}

Not applicable

\section{Authors' contributions}

Li Y, Jia J, and Mi Q carried out the overall experiments and analyzed the data. Li Y, and Gao Y collected the tissue samples during the SBDS disease outbreak in cherry valley duck farms. Li Y, Jia J wrote the paper. Zhu G helped to analyze the result and contributed to language polishing. Wang $\mathrm{J}$ conceived the project and directed the research.

\section{Acknowledgements}


This work was supported by the National Natural Science Foundation of China [grant numbers 31572551, 31172317] and by a project funded by the Priority Academic Program Development of Jiangsu Higher Education Institutions.

\section{References}

1. Chen H, Dou Y, Tang Y, Zhang Z, Zheng X, Niu X, Yang J, Yu X, Diao Y (2015) Isolation and genomic characterization of a duck-origin GPV-related parvovirus from cherry valley ducklings in China. Plos One 10(10):e0140284

2. Chen H, Dou Y, Tang Y, Zheng X, Niu X, Yang J, Yu X, Diao Y (2016) Experimental reproduction of beak atrophy and dwarfism syndrome by infection in cherry valley ducklings with a novel goose parvovirus-related parvovirus. Vet Microbiol 183:16-20

3. Cotmore SF, Agbandje-McKenna M, Chiorini JA, Mukha DV, Pintel DJ, Qiu J, Soderlund-Venermo M, Tattersall P, Tijssen P, Gatherer D, Davison AJ (2014) The family Parvoviridae. Arch Virol 159:12391247

4. Deng X, Dong Y, Yi Q, Huang Y, Zhao D, Yang Y, Tijssen P, Qiu J, Liu K, Li Y (2013) The determinants for the enzyme activity of human parvovirus B19 phospholipase A2 (PLA2) and its influence on cultured cells. Plos One 8(4):e61440

5. Derzsy D (1967) A viral disease of goslings. I. Epidemiological, clinical, pathological and etiological studies. Acta Vet Acad Sci Hungar 17:443-448

6. Fang D, Wang Y, Zhen Y, Zhou Y, Jiang M, Dong G (1981) Studies on the aetiology and specific control of gosling plaque. Scientia Agricultura Sinica (4):1-9 (in Chinese)

7. Gall-Reculé G L, Jestin V (1994) Biochemical and genomic characterization of Muscovy duck parvovirus. Arch Virol 139:121-131

8. Girod A, Wobus CE, Zádori Z, Ried M, Leike K, Tijssen P, Kleinschmidt JA, Hallek M (2002) The VP1 capsid protein of adeno-associated virus type 2 is carrying a phospholipase $A 2$ domain required for virus infectivity. J Gen Virol 83:973-978.

9. Kumar S, Stecher G, and Tamura K (2016) MEGA7: Molecular evolutionary genetics analysis version 7.0 for bigger datasets. Molecular Biology and Evolution 33:1870-1874

10. Li L, Qiu J, Pintel DJ (2009) The choice of translation initiation site of the rep proteins from goose parvovirus P9-generated mRNA is governed by splicing and the nature of the excised intron. $J$ Virol 83(19):10264-10268

11. Ning K, Liang T, Wang M, Dong Y, Qu S, Zhang D (2017) Genetic detection and characterization of goose parvovirus: Implications for epidemiology and pathogenicity in cherry valley Pekin ducks. Infect Genet Evol 51:101-103

12. Ning K, Liang T, Wang M, Dong Y, Qu S, Zhang D (2018) Pathogenicity of a variant goose parvovirus, from short beak and dwarfism syndrome of Pekin ducks, in goose embryos and goslings. Avian Pathol 47:391-399 
13. Niskanen E, Kalliolinna O, Ihalainen T, Häkkinen M, Vihinen-Ranta M. (2013) Mutations in DNA binding and transactivation domains affect the dynamics of parvovirus NS1 Protein. J Virol 87(21):11762-11774

14. Palya V, Zolnai A, Benyeda Z, Kovács E, Kardi V, Mató T (2009) Short beak and dwarfism syndrome of mule duck is caused by a distinct lineage of goose parvovirus. Avian Pathol 38:175-180

15. Palya VJ (2013) Parvovirus Infections of Waterfowl. In DE Swayne, JR Glisson, LR Mcdougald, LK Nolan, DL Suarez, V Nair. Diseases of Poultry, 13th edn (pp.444-451). Ames: Wiley-Blackwell.

16. Qiu J, Cheng F, Yoto Y, Zadori Z, Pintel D (2005) The expression strategy of goose parvovirus exhibits features of both the dependovirus and parvovirus genera. J Virol 79(17):11035-11044

17. Reed LJ, Müench H (1938). A simple method for estimating fifty percent endpoints. American Journal of Hygiene 27:493-497

18. Tewary SK, Zhao H, Deng X, Qiu J, Tang L (2014) The human parvovirus B19 non-structural protein 1 $\mathrm{N}$-terminal domain specifically binds to the origin of replication in the viral DNA. Virology 449:297303

19. Wang J, Duan J, Zhu L, Jiang Z, Zhu G (2015) Sequencing and generation of an infectious clone of the pathogenic goose parvovirus strain LH. Arch Virol 160(3):711-718

20. Yu K, Ma X, Sheng Z, Qi L, Liu C, Wang D, Huang B, Li F, Song M (2016) Identification of goose-origin parvovirus as a cause of newly emerging beak atrophy and dwarfism syndrome in ducklings. $\mathrm{J}$ Clin Microbiol 54:1999-2007

21. Zádori Z, Stefancsik R, Rauch T, Kisary J (1995) Analysis of the complete nucleotide sequences of goose and muscovy duck parvoviruses indicates common ancestral origin with adeno-associated virus 2. Virology 212:562-573

22. Zádori Z, Szelei J, Lacoste MC, Li Y, Gariépy S, Raymond P, Allaire M, Nabi IR, Tijssen P (2001) A viral phospholipase A2 is required for parvovirus infectivity. Dev Cell 1:291-302

\section{Tables}

Table 1 Primers designed for PCR amplification of the whole genomes of the NGPV isolates 


\begin{tabular}{|c|c|c|c|}
\hline Primer numbers & Primer sequences $\left(5^{\prime} \rightarrow 3^{\prime}\right)$ & Primer positions & Fragment sizes (bp) \\
\hline ITR-1 & tcattggagggttcgttcgttcg & $1-23 ; 5024-5046$ & 188 \\
\hline ITR-2 & gcatgcgcgcgcggtcaacctaa & $168-188 ; 4859-4879$ & \\
\hline ITR-3 & gcatgcgcgcggtcagcccaa & $183-203$ & 476 \\
\hline ITR-4 & ctcccaaactccggtagaaacc & $622-644$ & \\
\hline P1 & atggcactttctaggcctct & $507-526$ & 1378 \\
\hline P2 & aagaaggctccaatttatgtga & $1863-1884$ & \\
\hline P3 & gtattgaacctactcctgtaatt & $1741-1763$ & 1428 \\
\hline P4 & tgtagatgtggttgttgtagc & $3148-3168$ & \\
\hline P5 & ccgatggagtgggtaatgcct & $3049-3069$ & 1550 \\
\hline P6 & ttgagttagatatctggttccaat & $4575-4598$ & \\
\hline ITR-5 & ggccagtgtacagtagagatggtg & $4425-4448$ & 440 \\
\hline ITR-3 & gcatgcgcgcggtcagcccaa & $4844-4864$ & \\
\hline
\end{tabular}

Table 2 Background of the classical and novel goose parvovirus strains used for sequence comparison and phylogenetic analysis 


\begin{tabular}{|c|c|c|c|c|c|}
\hline Strain & Host & $\begin{array}{l}\text { Geographic } \\
\text { origin }\end{array}$ & $\begin{array}{l}\text { Isolation } \\
\text { year }\end{array}$ & Pathogenicity & $\begin{array}{l}\text { GenBank accession } \\
\text { number }\end{array}$ \\
\hline B & Goose & Hungary & 1967 & Pathogenic & U25749 \\
\hline VG32/1 & Goose & Germany & n.a. & Vaccine & EU583392 \\
\hline YZ99-6 & Goose & China & 1999 & Pathogenic & KC996730 \\
\hline LH & Goose & China & 2003 & Pathogenic & KM272560 \\
\hline $82-0321$ & Goose & China & 1982 & Pathogenic & EU583390 \\
\hline $06-0329$ & Goose & China & 2006 & Pathogenic & EU583391 \\
\hline SDLC01 & $\begin{array}{l}\text { Cherry valley } \\
\text { duck }\end{array}$ & China & 2015 & $\begin{array}{l}\text { Low } \\
\text { pathogenic }\end{array}$ & KT343253 \\
\hline JS1 & $\begin{array}{l}\text { Cherry valley } \\
\text { duck }\end{array}$ & China & 2016 & $\begin{array}{l}\text { Low } \\
\text { pathogenic }\end{array}$ & KT935536 \\
\hline QH15 & $\begin{array}{l}\text { Cherry valley } \\
\text { duck }\end{array}$ & China & 2015 & $\begin{array}{l}\text { Low } \\
\text { pathogenic }\end{array}$ & KT751090 \\
\hline SDHT16 & $\begin{array}{l}\text { Cherry valley } \\
\text { duck }\end{array}$ & China & 2016 & $\begin{array}{l}\text { Low } \\
\text { pathogenic }\end{array}$ & MN356043 \\
\hline SDJN19 & $\begin{array}{l}\text { Cherry valley } \\
\text { duck }\end{array}$ & China & 2019 & $\begin{array}{l}\text { Low } \\
\text { pathogenic }\end{array}$ & MN356044 \\
\hline SDLC19 & $\begin{array}{l}\text { Cherry valley } \\
\text { duck }\end{array}$ & China & 2019 & $\begin{array}{l}\text { Low } \\
\text { pathogenic }\end{array}$ & MN356045 \\
\hline D146/02 & Mule duck & France & 2002 & $\begin{array}{l}\text { Low } \\
\text { pathogenic }\end{array}$ & AY496906 \\
\hline D697/3/06 & Mule duck & France & 2006 & $\begin{array}{l}\text { Low } \\
\text { pathogenic }\end{array}$ & EU938706 \\
\hline
\end{tabular}

n.a.: not available

Due to technical limitations, table 3,4 is only available as a download in the Supplemental Files section.

\section{Figures}



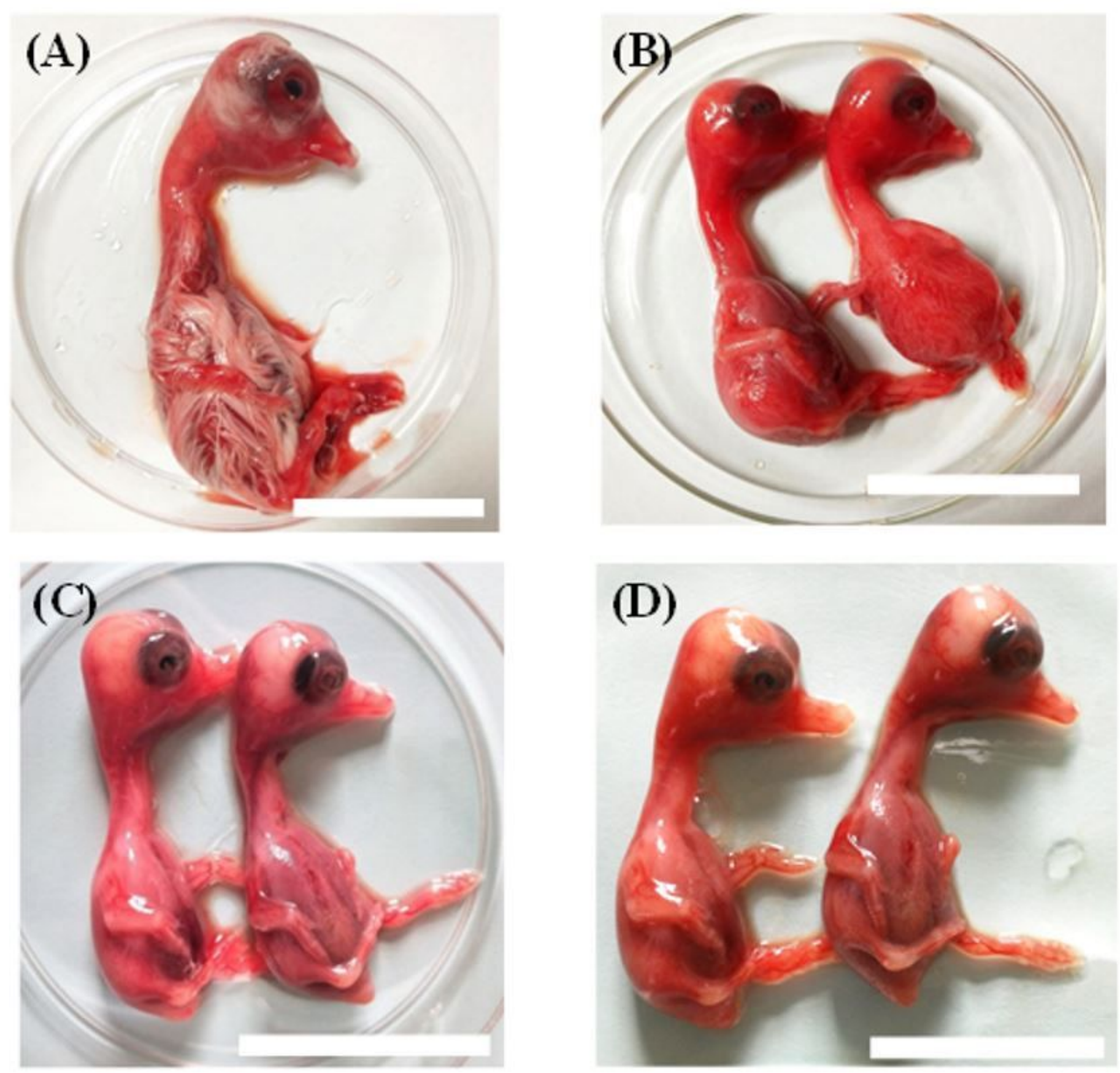

\section{Figure 1}

Hemorrhagic lesions were observed in goose or duck embryos that died at different time points postinfection during the isolation and passage stage of strain SDJN19 and SDHT16. (A) Goose embryos died at $264 \mathrm{~h}$ post-inoculation of the tissue sample homogenate. (B) Goose embryos died at $112 \mathrm{~h}$ after the first passage with the SDJN19 isolate. (B) Cherry valley duck embryos died at $96 \mathrm{~h}$ post-infection of the SDJN19 isolate. (D) Cherry valley duck embryos died at $112 \mathrm{~h}$ post-infection of the SDHT16 isolate. The scale bar is $30 \mathrm{~mm}$. 


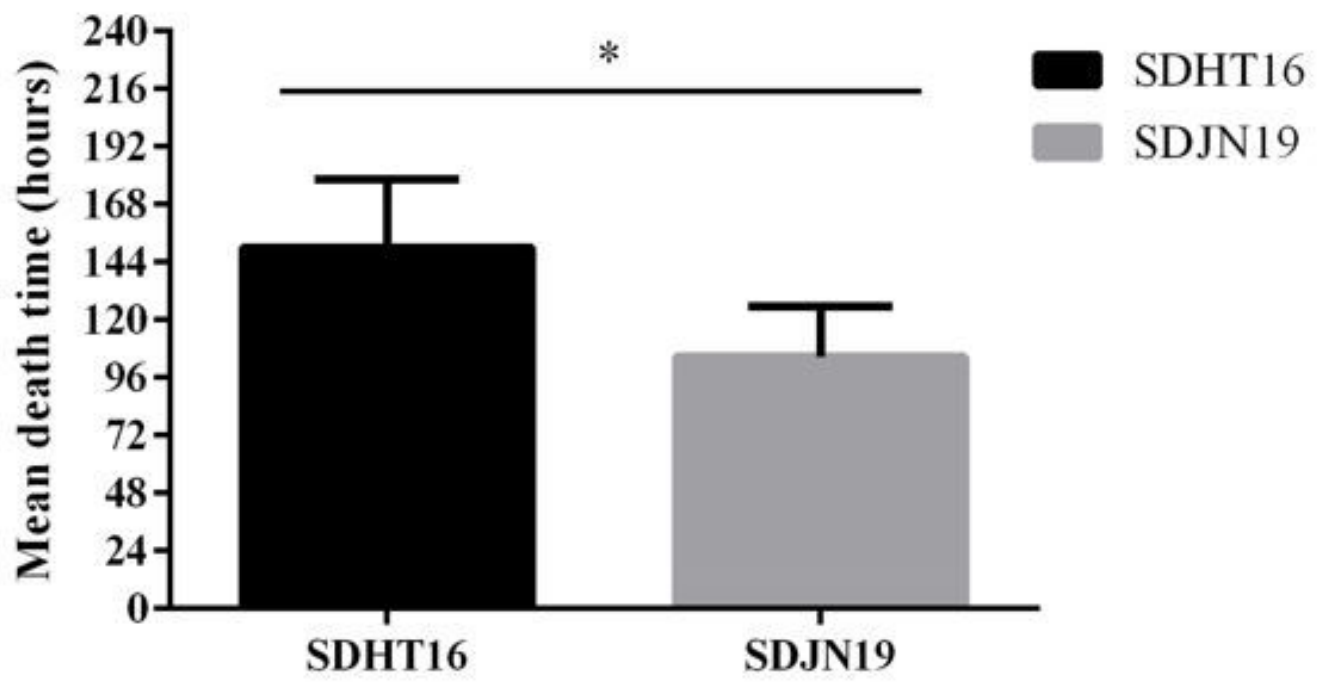

Figure 2

Comparison of the mean death time of the embryonated cherry valley duck eggs post-infection of strain SDHT16 and SDJN19.* Significant difference between SDHT16 and SDJN19-infected groups $(P<0.05)$.

Percent identity of VP1

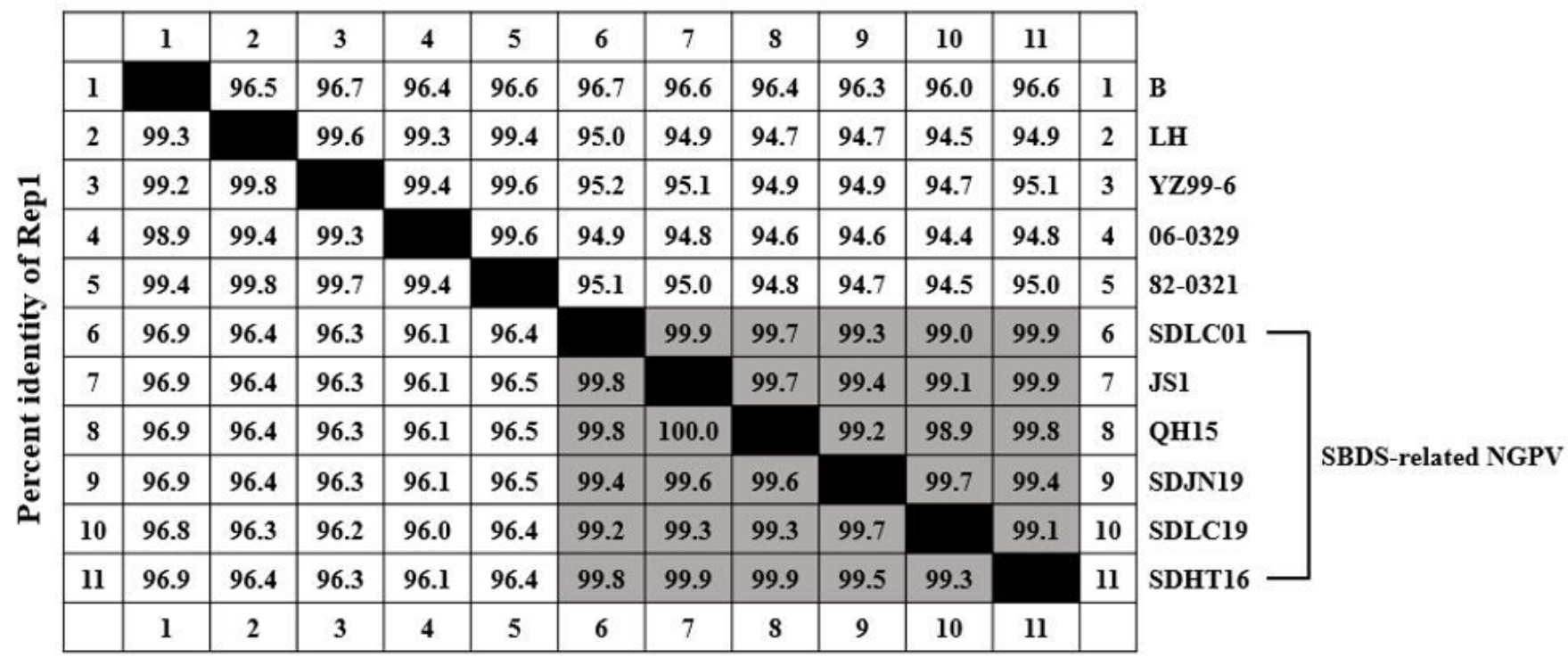




\section{Figure 3}

Percentages of the identities shared by the nucleotide sequences of the coding proteins VP1 (upper right) and Rep1 (lower left) of six NGPV strains and five classical GPV strains. Percentages of identities among the six NGPV strains are shaded in gray.

\begin{tabular}{|c|c|c|}
\hline LH & TCATTGGAGGGTTCGTTCGTTCGAACCAGCCAATCAGGGGAGGGGG-AAGTGACGCAAGTTCCGGTGACGCACATCCGGT & 79 \\
\hline DHT 16 & TTATTGGAGGGTTCGTTCGTTCGAGCCAGCCAATCAGGGGAGGGGG-AAGTGACGCAAGTCCCGGTCACGTGCTTCCGGT & 79 \\
\hline S JN 19 & TTATTGGAGGGTTCGTTCÄTTCGAGCCAGCCAATCAGGGGAGGGGGG̈AGTGACGCAAGTTCCGGTGACGTGCTTCCGGT & 80 \\
\hline SDLC19 & TIATTGGAGGGTTCGTTCATTCGAGCCAGCCAATCAGGGGAGGGGGGAGTGACGCAAGTTCCGGTGACGTGCITCCGGT & 80 \\
\hline LH & GACGTAGTTCCGGTCACGTGCTTCCTGTCACG 113 TITTCCGGTCACGTGACTTCCGGTCATGTGACTTCCGGTGACGTGT & 158 \\
\hline DHT 16 & GACGCACATCCGGTGACGTGCTTCCTGTCACGTGACTTCCGGTCATGTGACTTCCGGTCATGTGACTTCCGGTGACGTGT & 159 \\
\hline SDJN 19 & GACGCACATCCGGTCACGTGCTTCCTGTCACGTGACTTCCGGTCATGTGACTTCCGGTCATGTGACTTCCGGTGACGTGT & 160 \\
\hline SDLC19 & GACGCACATCCGGTCACGTGCTTCCTGTCACGTGACTTCCGGTCAIGTGACTTCCGGTCATGTGACTTCCGGTGACGTGT & 160 \\
\hline & Bubble region & \\
\hline . & TTCCGGCTGTTAGGTTGACCGCGCGCATGCGCGCGGTCAGCCCAATAGT TAAGCCGGAAACACGTCACCGGAAGTCACAT & 238 \\
\hline SDHT 16 & TTCCGGCTGTTAGGTTGACCĀCGCGCATGCGCGTGGTCAGCCCAATAGTTAAGCCGGAAACACGTCACCGGAAGTCACAT & 239 \\
\hline SDJN 19 & TTCCGGCTGTTAGGTTGACCACGCGCATGCGCGTGGTCAGCCCAATAGTTAAGCCGGAAACACGTCACCGGAAGTCACAT & 240 \\
\hline SDLC19 & TTCCGGCTGTTAGGTTGACCACGCGCATGCGCGIGGTCAGCCCAATAGTTAAGCCGGAAACACGTCACCGGAAGTCACAT & 240 \\
\hline LH & GACCGGAAGTCACGTGACCGGAAA-CACGTGACAGGAAGCACGTGACCGGAACTACGTCACCGGATGTGCGTCACCGGAA & 317 \\
\hline SDHT 16 & GACCGGAAGTCACA TGACCGGAAGTCACGTGACAGGAAGCACGTCACCGGATGTGCGTCACCGGA & 319 \\
\hline SDJN 19 & GACCGGAAGTCACATGACCGGAAGTCACGTGACAGGAAGCACGTGACCGGATGTGCGTCACCGGAAGCACGTCACCGGAA & 320 \\
\hline SDLC19 & GACCGGAAGTCACATGACCGGAAGTCACGTGACAGGAAGCACGTGACCGGATGTGCGTCACCGGAAGCACGTCACCGGAA & 320 \\
\hline & D sequence & \\
\hline LH & CTTGCGTCACTTCCCCC-TCCCCTGATTGGCTGGTTCGAACGAACGAACCCTCCAATGAGACTCAAGGACAAGAGGATAT & 396 \\
\hline SDHT 16 & CTTGCGTCACTTCCCCC-TCCCCTGATTGGCTGGCTCGAACGAACGAACCCTCCAATAAGACTCAAGGACAAGAAAATAT & 398 \\
\hline SDJN 19 & CTTGCGTCACTTCCCCCCTCCCCTGATTGGCTGGCTCGAATGAACGAACCCTCCAATAAGACTCAAGGACAAGAAAATAT & 400 \\
\hline SDLC19 & CTTGCGTCACTTCCCCCCTCCCCTGATTGGCTGGCTCGAATGAACGAACCCTCCAATAAGACTCAAGGACAAGAAAATAT & 400 \\
\hline LH & TTTGCGCGCCAGGAAGTG & 414 \\
\hline SDHT 16 & TTTGCGCGCCAGGAAGTG & 416 \\
\hline SDJN 19 & TTTGCGCGCCAGGAAGTG & 418 \\
\hline SDLC19 & TTTGCGCGCCAGGAAGTG & 418 \\
\hline
\end{tabular}

\section{Figure 4}

Nucleotide sequence alignment of ITRs among three NGPV strains (SDHT16, SDJN19, and SDLC19) and the classical GPV strain LH. The different nucleotides or insertions relative to the classical GPV strain LH are shaded in grey. The dashes denote deletions. The sequences in the bubble region of ITR are composed of 43 nucleotides and indicated by open circles above the letters. The $D$ sequences are underlined, which reversely complement with the D' sequences at the 3' ITR. 


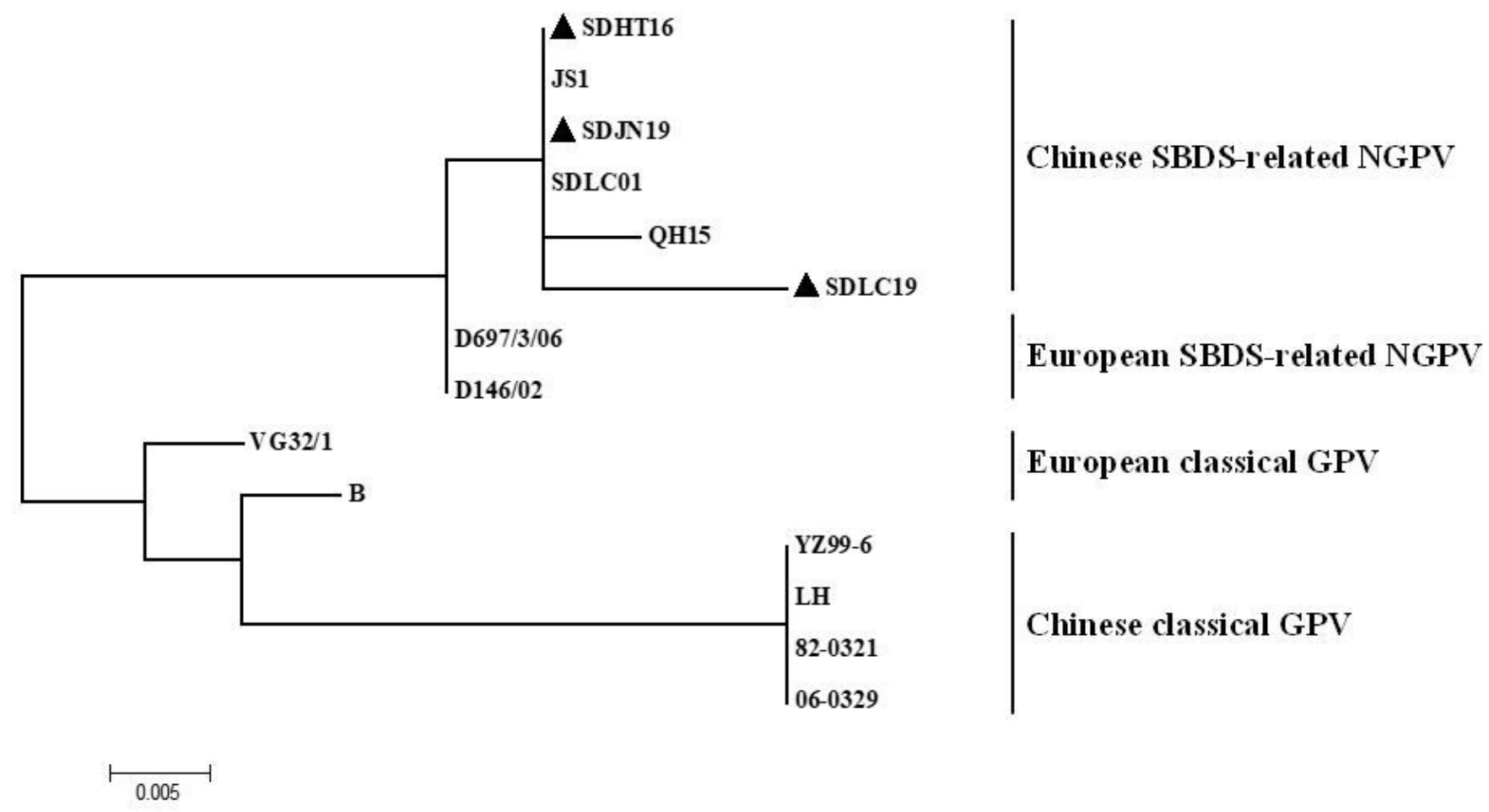

Figure 5

Phylogenetic analysis of the SBDS-related NGPV and the classical GPV strains. The phylogenetic tree was constructed on the basis of a 427-bp VP1 gene fragment. The Chinese SBDS-related NGPV strains clustered with the European NGPV strains, forming a separate branch. Phylogenetic trees were constructed through the neighbor-joining method in MEGA 7.0 with 1000 bootstrap replications.

\section{Supplementary Files}

This is a list of supplementary files associated with this preprint. Click to download.

- Table3.jpg

- Table4.jpg 